\title{
Pretty Printing with Lazy Dequeues
}

OLAF CHITIL

University of Kent, UK

There are several purely functional libraries for converting tree structured data into indented text, but they all make use of some backtracking. Over twenty years ago Oppen published a more efficient imperative implementation of a pretty printer. This paper shows that the same efficiency is also obtainable without destructive updates by developing a similar but purely functional Haskell implementation with the same complexity bounds. At its heart lie two lazy double ended queues.

Categories and Subject Descriptors: D.1.1 [Programming Techniques]: Applicative (Functional) Programming; E.1 [Data Structures]: Lists, Stacks, and Queues

General Terms: Algorithms, Languages

\section{PRETTY PRINTING}

Pretty printing is the task of converting tree structured data into text, such that the indentation of lines reflects the tree structure. Furthermore, to minimise the number of lines of the text, substructures are put on a single line as far as possible within a given line-width limit. Here is the result of pretty printing an expression within a width of 35 characters:

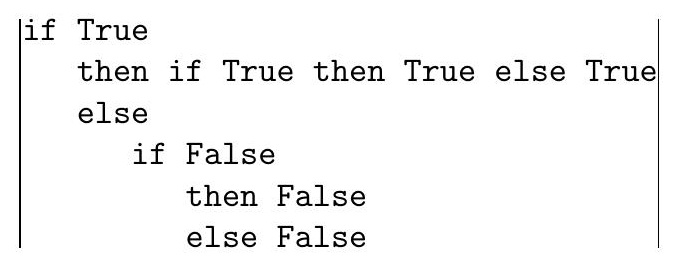

John Hughes [1995], Simon Peyton Jones [1997], Phil Wadler [2003], and Pablo Azero and Doaitse Swierstra [1998] have all developed pretty printing libraries for the functional language Haskell [Peyton Jones 2003]. Such a library implements the functionality common to a large class of pretty printers. For example, Wadler's library provides the following functions:

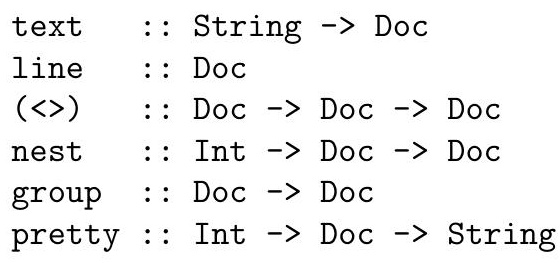

Permission to make digital/hard copy of all or part of this material without fee for personal or classroom use provided that the copies are not made or distributed for profit or commercial advantage, the ACM copyright/server notice, the title of the publication, and its date appear, and notice is given that copying is by permission of the ACM, Inc. To copy otherwise, to republish, to post on servers, or to redistribute to lists requires prior specific permission and/or a fee. 
The function text converts a string to an atomic document, the document line denotes a (potential) line break, and <> concatenates two documents. The function nest increases the indentation for all line breaks within its document argument. The function group marks the document as a unit to be formatted either horizontally, that is on a single line by converting each line break (and corresponding indentation) into a single space, or vertically, with all line breaks unchanged. Finally, the function pretty yields a string with a pretty layout. The function aims at minimising the number of lines by formatting groups horizontally while not exceeding the given line-width limit. The layout of a subdocument depends not only on its form but also on its context, the remaining document. The library functions enable easy compositional construction of a document

To pretty print a concrete data structure, we only have to define a function that transforms the data structure into a document. With the following function we obtain the pretty printed expression shown on the previous page:

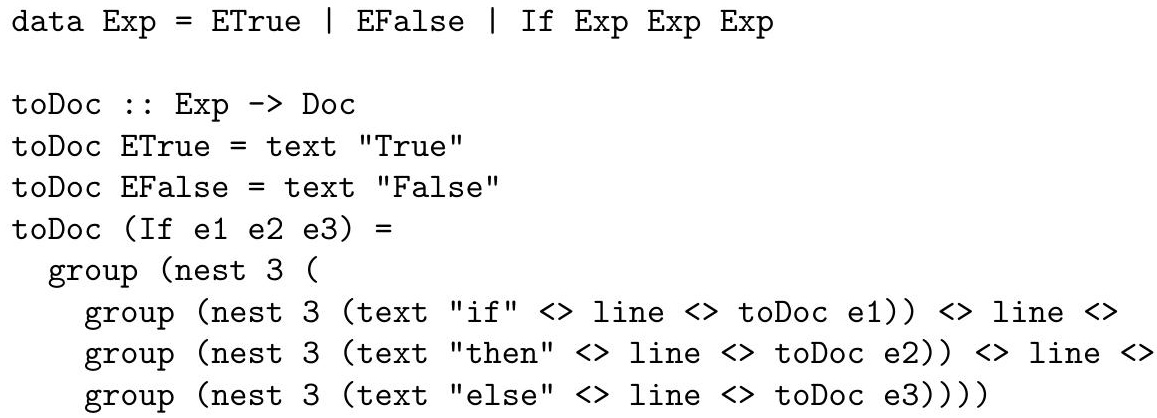

All previous implementations of Haskell pretty printing libraries use backtracking to determine the optimal layout. They limit backtracking to achieve reasonable efficiency, but their time complexity is worse than linear in the size of the input. However, more than 20 years ago Dereck Oppen [1980] published an imperative implementation of a pretty printer with linear time complexity. At the heart of his implementation lies an array that is updated in a complex pattern. Wadler tried to translate this implementation into a functional language but did not succeed [Wadler 2003]. Are destructive updates necessary to achieve efficiency? No, but the proof is not straightforward. We develop here, step by step, guided by Oppen's implementation, a similar but purely functional implementation in Haskell.

We implement Wadler's pretty printing interface. The interfaces of Hughes' and Peyton Jones' libraries are different, but changing the implementation to support them seems possible. Because Azero's and Swierstra's library is more expressive than the others, the implementation cannot support its interface.

\section{THE PROBLEM}

We follow Wadler in considering a document of type Doc as equivalent to a set of strings with the same content but different layout. These are the strings that we obtain for all the possible choices of formating the groups occurring in the construction of the document. Every group can be formatted independently either horizontally or vertically, except that all groups within a horizontal group have 
to be horizontal as well. The function pretty chooses one member of the set of strings.

To specify the output of pretty, we define a document as an algebraic data type with a constructor for each function that yields a document:

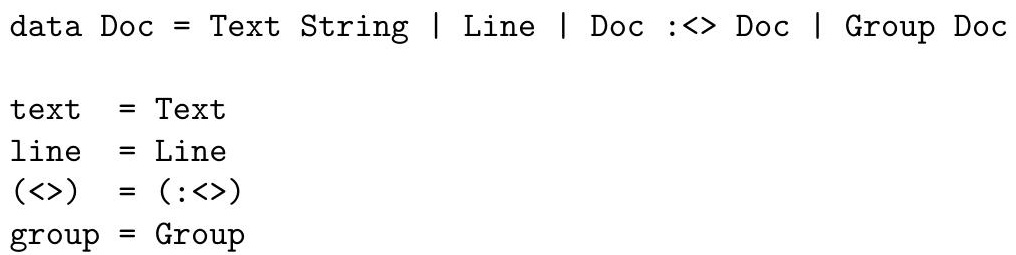

For simplicity we ignore the function nest for the moment. We will see in Section 8 how it can easily be added to the final implementation.

We use a "document interpreter" inter to define the function pretty. While recursively traversing the document in-order, the function inter keeps track of two state variables: the boolean $\mathrm{h}$ states if the interpreter is within a horizontal group; the integer $r$ contains the size of the remaining space on the current line. Besides the formatted output, the interpreter also has to return the remaining space on the last line.

For formatting a group the interpreter takes Oppen's approach: a group is formatted horizontally if and only if it fits on the remaining space of the line. In Section 7 this choice is discussed in more detail. A function fits checks if the remaining space is sufficient. ${ }^{1}$

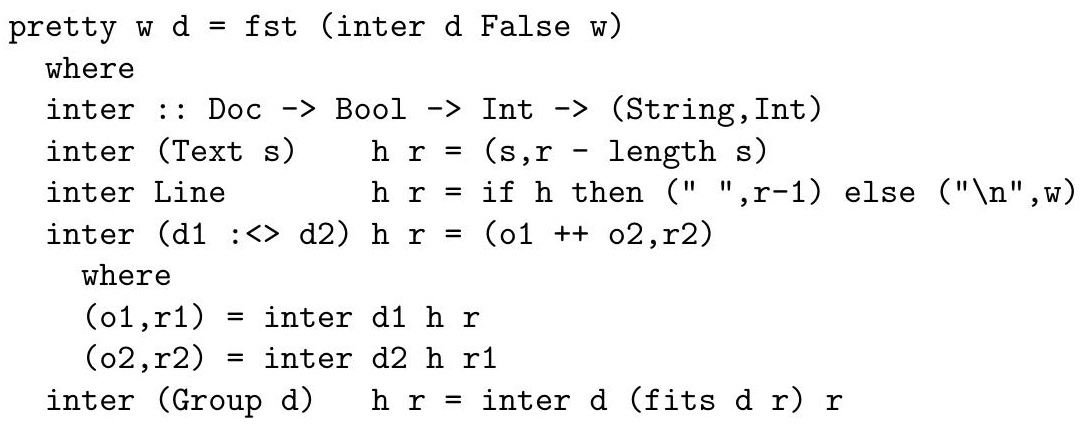

A naïve implementation of fits evaluates the width of the document $d$ and compares the result with the remaining space $r$.

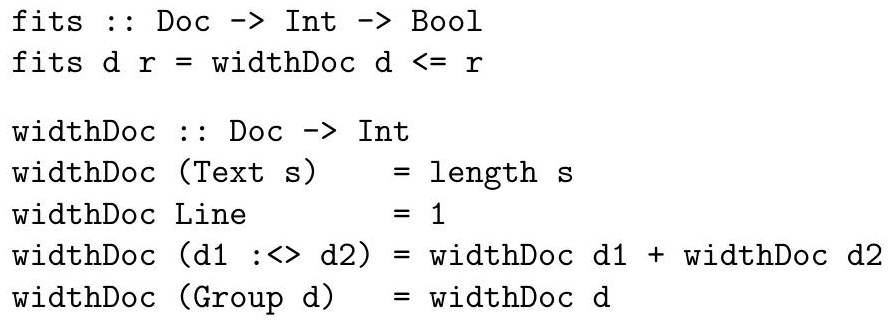

${ }^{1}$ Because a group within a horizontal group is horizontal, we could replace the boolean expression fits $d r$ by $h$ ll fits $d r$ in the last equation of inter. This optimisation, however, does not improve the time complexity of this implementation nor any other one presented later.

ACM Transactions on Programming Languages and Systems, Vol. TBD, No. TDB, Month Year. 
The naïve implementation has the disadvantage that the additional traversals of the groups to determine their widths causes the function pretty to require exponential time for formatting some documents with nested groups.

There is another problem: Only after the full traversal of a group it is known whether the group fits on the remaining line. Hence the interpreter produces most of the output string for a group only after it has traversed the whole group, as the following computation of the Haskell interpreter Hugs ${ }^{2}$ demonstrates:

Main> pretty 4 (group (text "Hi" <> line <> text "you" \&> undefined)) "Hi

Program error: undefined

So the document for a whole group has to be kept in memory. A group is often large; it may encompass the whole document. Furthermore, the document itself can often be constructed lazily and hence should never exist as a whole in memory. In interactive applications the time delay at the beginning of a group may be disturbing. A pretty printer should only require a look-ahead of at most $w$ characters, were $w$ is the line-width limit. Wadler [2003] calls an implementation with this property bounded.

\subsection{Bounded but not Linear}

We can define fits so that it traverses the document $d$ at most up to the remaining width $r$. When that point is reached, it is clear that the document does not fit.

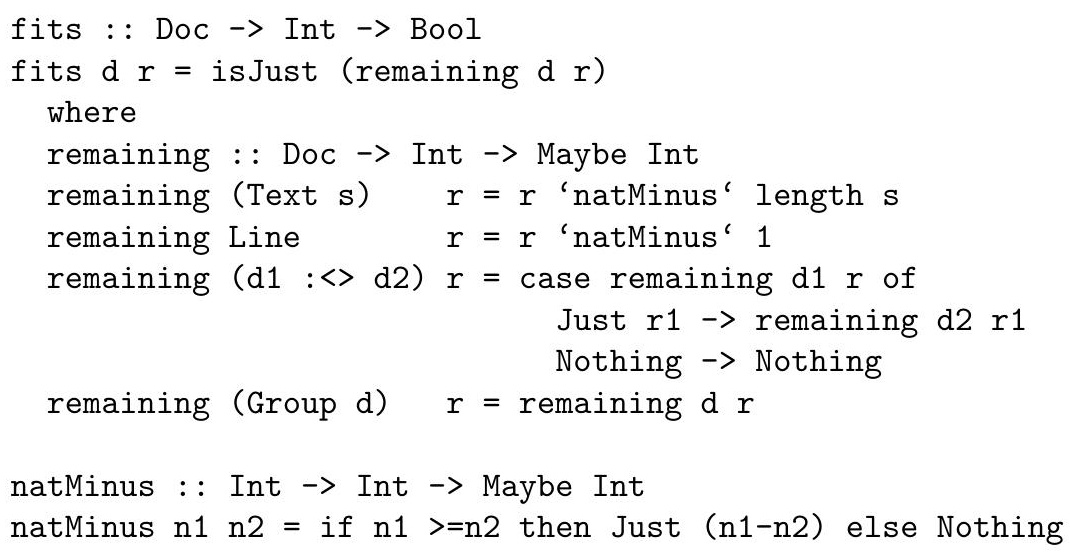

This implementation with pruning is bounded:

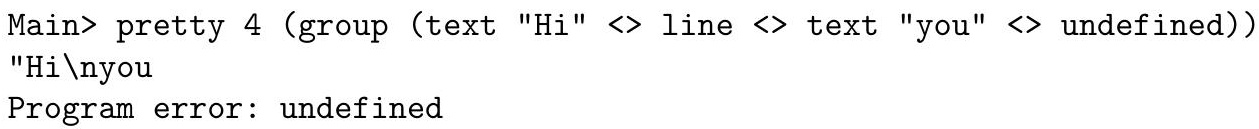

The pruning method is similar to Wadler's method of pruning backtracking and hence we obtain the same time complexity: In the worst case it is $O(n \cdot w)$, where $n$ is the size of the input and $w$ the line-width limit. An example for the worst case is the following right-nested document:

\footnotetext{
${ }^{2}$ http://www.haskell.org/hugs

ACM Transactions on Programming Languages and Systems, Vol. TBD, No. TDB, Month Year.
} 
group (text $" * "<>$ line $<>$ group (text $" * "<>$ line $<>$ group ( . . )))

Pruning substantially improves the time complexity, but we want to obtain $O(n)$ time complexity, independent of $w$. However, no further optimisation is in sight. The optimisation leads into a cul-de-sac.

\subsection{Linear but not Bounded}

On the other hand, we can obtain a linear implementation from the naïve definition by tupling the document traversals and creating a circular program [Bird 1984]: instead of a separate function that traverses a document to determine its width, the interpreter inter can determine the width in addition to its other tasks. The new version of inter returns the formatted document, the size of the space remaining on the last line, and the width of the document:

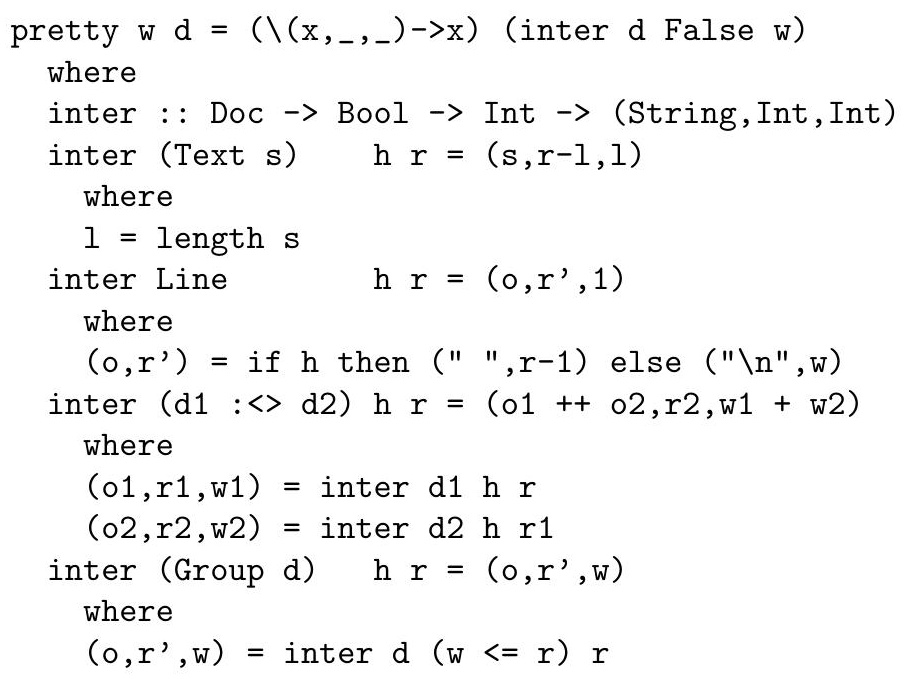

This implementation of inter takes advantage of lazy evaluation: in the last equation the result width $\mathrm{w}$ is passed as part of the second argument. For this circular definition to work, the function inter has to be able to yield the width of a document without using the value of $\mathrm{h}$. Therefore it would be wrong to "simplify" the equation for Line to

inter Line $\mathrm{h} r=$ if $\mathrm{h}$ then (" ", $\mathrm{r}-1,1$ ) else ("\n",w,1)

The implementation has linear time complexity ${ }^{3}$, because a computation spends only constant time on each document constructor. However, the implementation is not bounded.

\subsection{Recapitulation}

This section has set the scene. We specified the meaning of the pretty printing functions and stated the two desired properties of boundedness and linear time

\footnotetext{
3 The use of $(++)$ for formatting a document $\mathrm{d} 1$ : $<>$ d2 actually leads to quadratic time complexity. To achieve linear time we can represent a document as a function and then function composition performs list concatenation [Hughes 1986]. We do not apply this optimisation here to not to distract from the main issues.
}

ACM Transactions on Programming Languages and Systems, Vol. TBD, No. TDB, Month Year. 
complexity. There are implementations that are bounded and others that are linear. The challenge is to marry the two properties in a single implementation.

\section{A LINEAR IMPLEMENTATION WITH STACKS}

The tree structured recursion of inter and pruning at a certain width to achieve boundedness do not fit together. Hence we follow Oppen and represent a document not as a tree structure but as a token sequence.

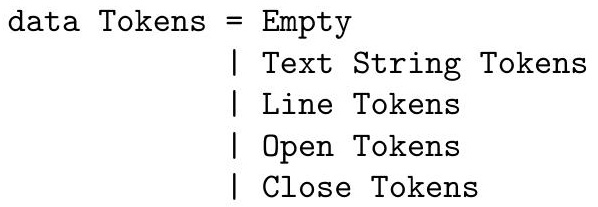

A group is represented as an Open token, the sequence of the grouped document and a final Close token. To construct the token sequence in linear time we represent a document as a function and function composition performs concatenation [Hughes 1986].

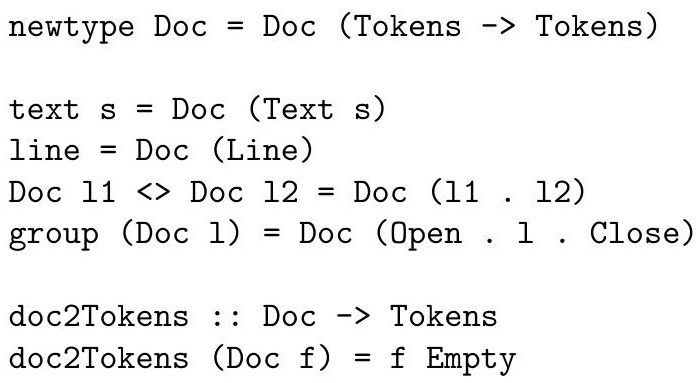

Similar to our previous implementations we define pretty through an interpreter inter of the token sequence. Because this interpreter iterates along the token list instead of recursively following the nesting structure of groups, it has to store information about surrounding groups explicitly in stacks. We will see that these explicit data structures are the key to obtaining the desired implementation.

We use the following abstract data type of sequences with stack operations:

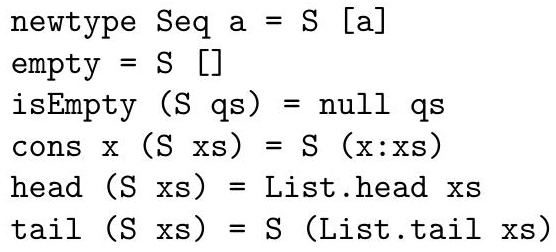

Using an abstract data type avoids premature commitment to the concrete representation. If we used lists directly we would also be tempted to use pattern matching in place of the functions isEmpty, head and tail, which would make later changes of representation even harder. Okasaki [2000] gives a compelling example that demonstrates how easily premature commitment to a representation can make functional programmers blind to a natural implementation solution. 


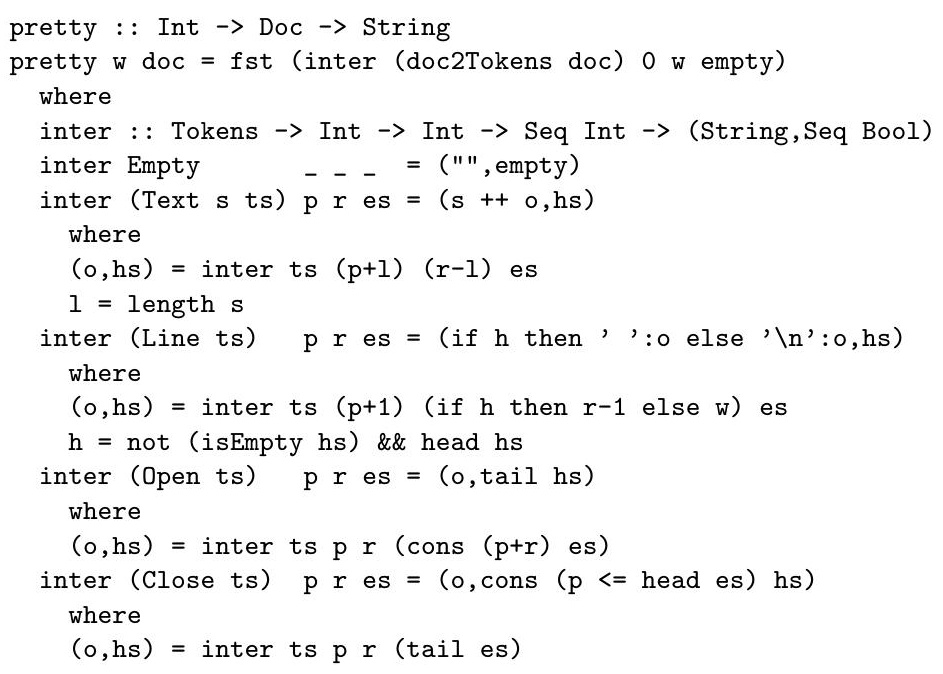

Fig. 1. Implementation using Stacks

For our first implementation of the token interpreter we do not yet care about boundedness. So the interpreter decides if a group is formatted horizontally or vertically only after it has been traversed, that is, the decision is made at the Close token of the group. An implementation that makes the decision by determining the width of a group proves to be rather complicated. Instead it is easier to follow Oppen again and introduce an absolute measure of a token's position. The absolute position $\mathrm{p}$ gives the column in which a token would start, if the whole document that is passed to pretty was formatted in a single line. The interpreter keeps track of the absolute position of the current token and the remaining space, as used already in the last section. At an Open token the interpreter adds absolute position and remaining space to determine the maximal end position of the group. If the maximal end position is larger or equal than the absolute position of the Close token of the group, then the group fits on the line and hence is formatted horizontally. Otherwise it is formatted vertically.

To get the maximal end position of a group from its Open token past possibly many inner groups to its Close token, the interpreter passes a stack of maximal end positions along the token sequence. Inversely, the interpreter has to get the information whether a group is horizontal from its Close token back to all its Line tokens, because these need to be formatted accordingly. For this purpose the interpreter passes a stack of boolean values, called the horizontal stack, back along the token sequence.

Figure 1 shows the implementation. As the type of inter indicates, it passes the arguments $\mathrm{p}$ (absolute position), $\mathrm{r}$ (remaining space) and es (stack of maximal end positions) along the token sequence, whereas it returns o (output) and hs (horizontal stack) in the opposite direction. At an Open token an element is pushed on the stack of maximal end positions and the top element from the horizontal stack is popped. At a Close token the top element of the stack of maximal end positions is popped and the decision on formatting is pushed on the horizontal stack. Each

ACM Transactions on Programming Languages and Systems, Vol. TBD, No. TDB, Month Year. 
element of a stack corresponds to a surrounding group.

The subsequent table shows the values of the main interpreter variables for an example token list. We assume that the strings of the Text tokens have length 1. The line-width limit is 3 . Sequences are enclosed in \langle\rangle and boolean values are abbreviated as $\mathrm{T}$ and $\mathrm{F}$.

$$
\begin{aligned}
& \text { Open Text Line Open Text Line Text Close Close } \\
& \mathrm{p} \quad 0 \rightarrow 0 \rightarrow 1 \rightarrow 2 \rightarrow 2 \rightarrow 3 \rightarrow 4 \rightarrow 5 \rightarrow 5 \rightarrow 5 \\
& \mathrm{r} 3 \rightarrow 3 \rightarrow 2 \rightarrow 3 \rightarrow 3 \rightarrow 2 \rightarrow 1 \rightarrow 0 \rightarrow 0 \rightarrow 0 \\
& \text { es }\langle\rangle \rightarrow\langle 3\rangle \rightarrow\langle 3\rangle \rightarrow\langle 3\rangle \rightarrow\langle 5,3\rangle \rightarrow\langle 5,3\rangle \rightarrow\langle 5,3\rangle \rightarrow\langle 5,3\rangle \rightarrow\langle 3\rangle \rightarrow\langle\rangle \\
& \text { hs }\langle\rangle \leftarrow\langle\mathrm{F}\rangle \leftarrow\langle\mathrm{F}\rangle \leftarrow\langle\mathrm{F}\rangle \leftarrow\langle\mathrm{T}, \mathrm{F}\rangle \leftarrow\langle\mathrm{T}, \mathrm{F}\rangle \leftarrow\langle\mathrm{T}, \mathrm{F}\rangle \leftarrow\langle\mathrm{T}, \mathrm{F}\rangle \leftarrow\langle\mathrm{F}\rangle \leftarrow\langle\rangle
\end{aligned}
$$

The arrows indicate the direction in which values are passed along the token sequence. The table does not show the complex data dependencies between values. Similar to the last interpreter in the previous section this one is circular: in the interpreter equation for the token Line, information from the returned horizontal stack is passed as part of the third argument, the remaining space. For this to work the decision if a group is horizontal may not depend on the remaining space at its inner tokens. Indeed, the decision only depends on the remaining space at its Open token.

Laziness leads to a kind of co-routine computation. The computation of each passed value, that is, absolute position, remaining space, maximal end position stack, horizontal stack and output, can be identified with a process. At one point in time each process may be at a different position in the token sequence. The computation of the horizontal stack is furthest along the sequence, "looking" for Close tokens. The computation of the output is the most backward process, directly or indirectly using the results of all other processes.

For interpreting a token the implementation only requires time linear in the size of a token (the size of Text $s$ is the length of $s$ ). Hence with $n$ as the size of the input, the implementation has $O(n)$ time complexity.

\section{EARLIER DECISIONS THROUGH DEQUEUES}

The linear implementation with stacks is not bounded, because the interpreter decides whether a group is formatted horizontally only at the Close token of the group. However, in the example table of the previous section the interpreter could have noticed already at the second Line token that the outer group does not fit, because the absolute position 4 after the token is larger than the maximal end position of the group, the 3 stored at the rear of the sequence es before the token. The interpreter could have removed the maximal end position from the rear of the sequence and could have added a False to the rear of the corresponding horizontal sequence hs.

The following table shows the variable values for a modified implementation. Underlined values differ from those of the previous table.

$$
\begin{aligned}
& \text { Open Text Line Open Text Line Text Close Close } \\
& \mathrm{p} \quad 0 \rightarrow 0 \rightarrow 1 \rightarrow 2 \rightarrow 2 \rightarrow 3 \rightarrow 4 \rightarrow 5 \rightarrow 5 \rightarrow 5 \\
& \mathrm{r} 3 \rightarrow 3 \rightarrow 2 \rightarrow 3 \rightarrow 3 \rightarrow 2 \rightarrow 1 \rightarrow 0 \rightarrow 0 \rightarrow 0 \\
& \text { es }\langle\rangle \rightarrow\langle 3\rangle \rightarrow\langle 3\rangle \rightarrow\langle 3\rangle \rightarrow\langle 5,3\rangle \rightarrow\langle 5, \mathbf{3}\rangle \rightarrow\langle 5\rangle \rightarrow\langle 5\rangle \rightarrow\langle\rangle \rightarrow\langle\rangle \\
& \text { hs }\langle\rangle \leftarrow\langle\mathrm{F}\rangle \leftarrow\langle\mathrm{F}\rangle \leftarrow\langle\mathrm{F}\rangle \leftarrow\langle\mathrm{T}, \mathrm{F}\rangle \leftarrow\langle\mathrm{T}, \mathrm{F}\rangle \leftarrow \overline{\langle\mathrm{T}\rangle} \leftarrow \overline{\langle\mathrm{T}\rangle} \leftarrow \overline{\langle\rangle} \leftarrow\langle\rangle
\end{aligned}
$$

ACM Transactions on Programming Languages and Systems, Vol. TBD, No. TDB, Month Year. 
The modified interpreter removes elements from the rear of es and adds elements to the rear of hs as well as still adding and removing elements from the front. So the sequences es and hs are no longer used as stacks but as double ended queues (dequeues). For the moment we extend our sequence type accordingly in an inefficient but straightforward way:

$\operatorname{snoc} x(S x s)=S(x s++[x])$

last $(S \mathrm{xs})=$ List.last $\mathrm{xS}$

init $(S \mathrm{xS})=\mathrm{S}$ (List.init $\mathrm{xs}$ )

The general idea of earlier formatting decisions is as follows: every time the interpreter increases the absolute position, it checks if the maximal end position for the outermost surrounding group is smaller than the absolute position. If it is smaller, than that group has to be formatted vertically. So the interpreter removes the maximal end position from the rear of es and adds the boolean False to the rear of hs. The maximal end position is removed from the rear of es for two reasons. First, the new maximal end position at the rear of the sequence has to be compared with the current absolute position: the interpreter should be able to decide for several nested groups that they are vertical. Second, removal of the maximal end position ensures that the sequence is empty when the Close token of the group is reached and thus the interpreter notices at the Close token that the decision on the formatting of the group has been taken already.

As in the previous implementation, every element of a sequence es or hs contains information about a surrounding group. The front element corresponds to the directly surrounding group, the next element to the next surrounding group, etc. Only, now there may no longer be an element for every surrounding group. If there is no maximal end position for a group in es, then the maximal end position is smaller than the absolute position of the current token. If there is no "horizontal" boolean for a group in hs, then that group is formatted vertically.

Figure 2 shows the new implementation with es and hs as dequeues. The function prune implements the new early check. The definition is recursive, because at a given absolute position several surrounding groups may be found to require vertical formatting. The definition of inter differs from the previous one in that the cases for Text and Line tokens call the function prune, because these are the tokens that cause an increase of the absolute position. Because of earlier decisions, the maximal end positions sequence can already be empty at a Close token. In that case both sequences are unchanged. Otherwise a boolean is put in front of the horizontal sequence as before. ${ }^{4}$

This implementation is not linear, because of the inefficient new dequeue functions. The recursive definition of the function prune does not endanger linear runtime, because there is at most one recursive call of prune per Open token in the token list.

\footnotetext{
${ }^{4}$ We could replace the test $\mathrm{p}<=$ head es by True. The correctness of this minor optimisation relies, however, on es being ordered, with the largest element at the rear. When in Section 8 the function nest is added to the implementation, this ordering property will no longer hold.
} 


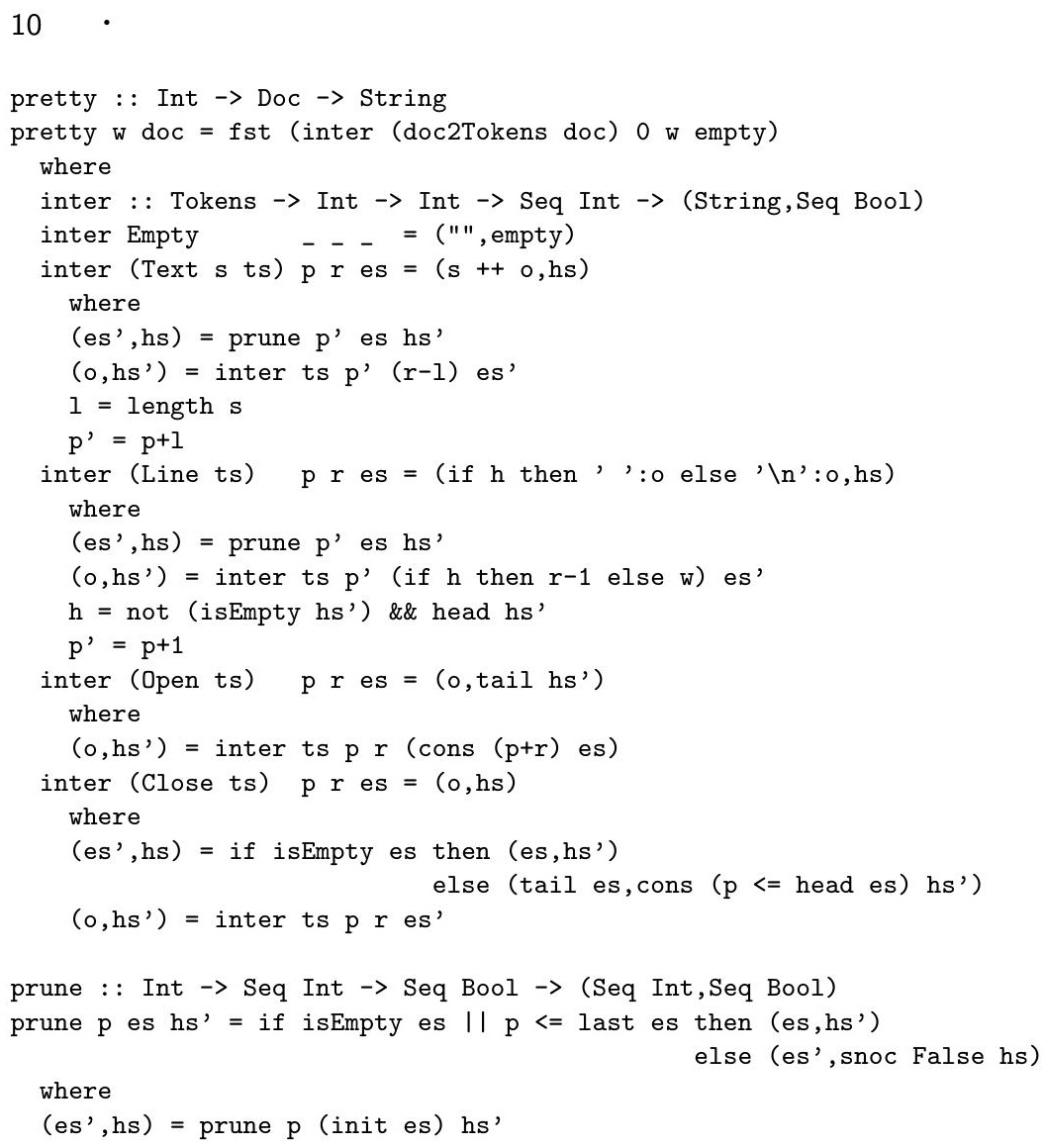

Fig. 2. Implementation using Dequeues

From a given Open token the new interpreter requires only a look-ahead of at most the width limit ${ }^{5}$ along the token sequence to decide if the group is formatted horizontally or vertically. However, despite the earlier decisions the implementation is still unbounded.

\section{LIMITING LOOK-AHEAD THROUGH LAZY DEQUEUES}

Why is the implementation with earlier decisions still unbounded? It is the fault of the functions applied to the horizontal sequence hs. On the one hand, when the need for vertical formatting is detected early, a False value is added to the rear of hs. On the other hand, at every Line token of the group, the front value of hs is used to decide the formatting. So the False "travels" from the rear to the front of the sequence. Evaluation of the front element of hs forces nearly full evaluation of hs.

\footnotetext{
${ }^{5}$ The look-ahead is also determined by the length of Text strings. Calling prune after every character of a Text string would remove this dependency, but increase the runtime considerably. In practice, Text strings should be shorter than the width limit; otherwise no "pretty" formatting is possible anyway.

ACM Transactions on Programming Languages and Systems, Vol. TBD, No. TDB, Month Year.
} 


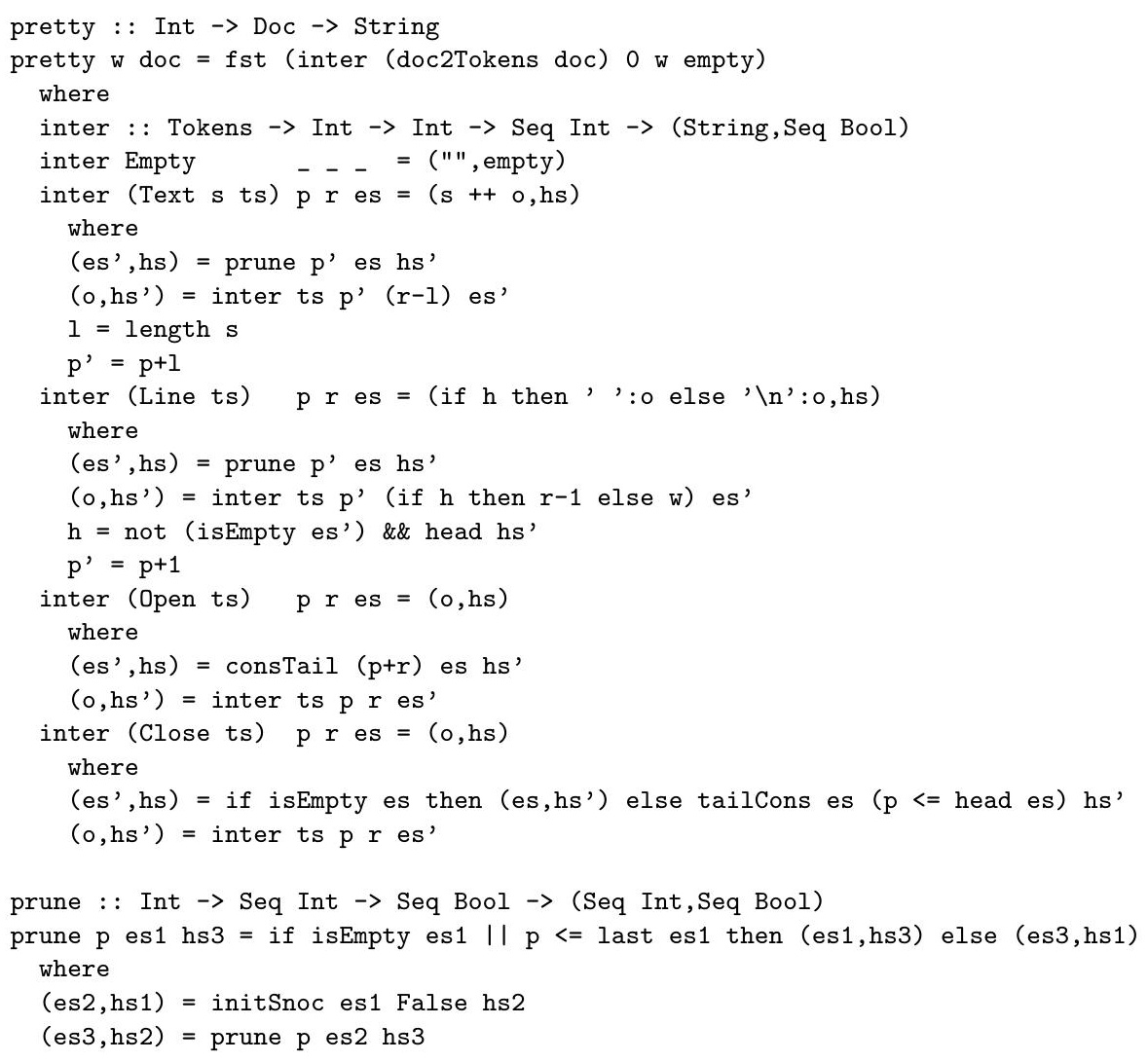

Fig. 3. Implementation using Combined Dequeue Functions

The following equations demonstrate the problem:

head (tail (snoc False (undefined))) = undefined

head (tail (snoc False $(S$ [undefined])) ) = False

head (tail (snoc False $(\mathrm{S}$ [True])) ) False

To determine the front element, the values of other sequence elements (e.g. True) are not needed, but the structure of the sequence, in particular its length, is.

The solution of the problem lies in the close relationship between the maximal end position sequence and the horizontal sequence. In the implementation of Figure 2 the two sequences are modified in perfect synchrony. At every program point where a function yielding a new sequence is applied to one sequence, the inverse function is applied to the other sequence. Hence we can combine the operations on two sequences. Let

consTail : : a $\rightarrow$ Seq a $\rightarrow$ Seq $b \rightarrow$ (Seq a, Seq b)

add an element to the front of the first sequence and take the tail of the second one;

tailCons : : Seq a $\rightarrow$ b $\rightarrow$ Seq $b \rightarrow$ (Seq a, Seq b)

ACM Transactions on Programming Languages and Systems, Vol. TBD, No. TDB, Month Year. 
take the tail of the first sequence and add an element to the front of the second sequence;

initSnoc : : Seq a $\rightarrow$ b $\rightarrow$ Seq $b \rightarrow$ (Seq a, Seq b)

take the initial part of the first sequence and add an element to the rear of the second sequence. The implementation in Figure 3 differs from that of Figure 2 only insofar that it uses the new combined functions on sequences and uses isEmpty es' instead of isEmpty hs'.

The sequence es is passed forwards along the token sequence and the sequence hs is passed backwards along the token sequence. Both sequences are empty at the beginning and at the end of the token sequence. Together with the perfect synchrony of modifying the two sequences follows that for any token of the token sequence both es and hs have the same length. Hence the interpreter can use the internal structure of es, which may be fully evaluated, to apply a function to hs without evaluating any part of hs.

We define an auxiliary function:

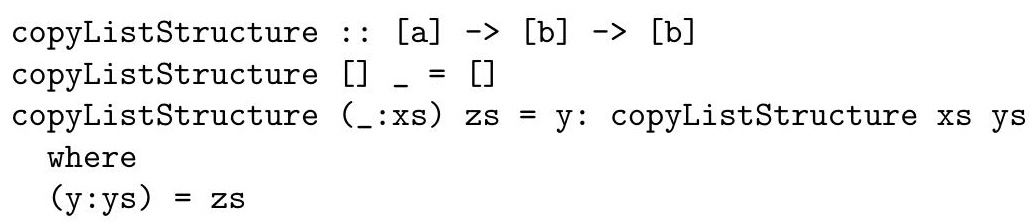

This function takes two lists that should be of the same length. The function makes a copy of the second list, using the structure of the first list. Thus the list structure of the result can be fully evaluated, without evaluating the second list at all. For example:

copyListStructure $[1,2,3]$ undefined = [undefined, undefined, undefined]

Using copyListStructure we define new lazy combined dequeue functions. Each combined function copies the structure of the first sequence (es) to the second sequence (hs) and only then applies the actual function to the copy.

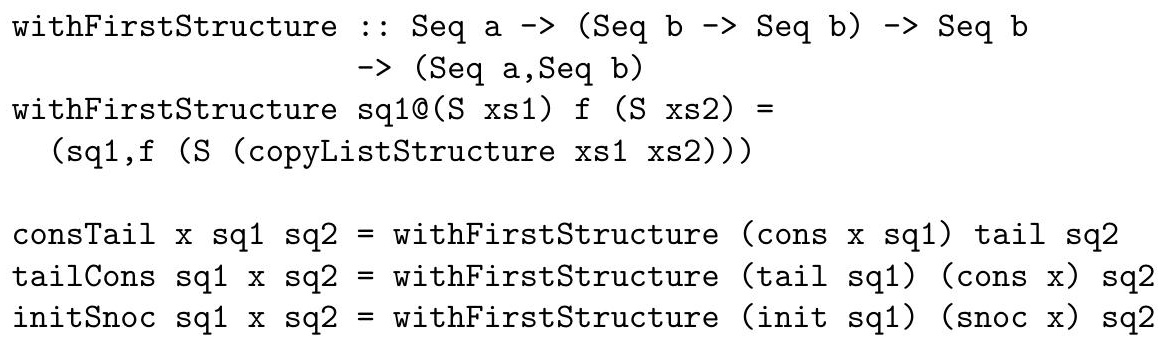

With these lazy dequeue functions the implementation of Figure 3 is bounded.

\section{EFFICIENT LAZY DEQUEUES}

In making the pretty printer bounded we have lost again the time efficiency of the first token list implementation. The functions snoc and last, and in particular copyListStructure take time linear in the length of the sequence argument. To ACM Transactions on Programming Languages and Systems, Vol. TBD, No. TDB, Month Year. 


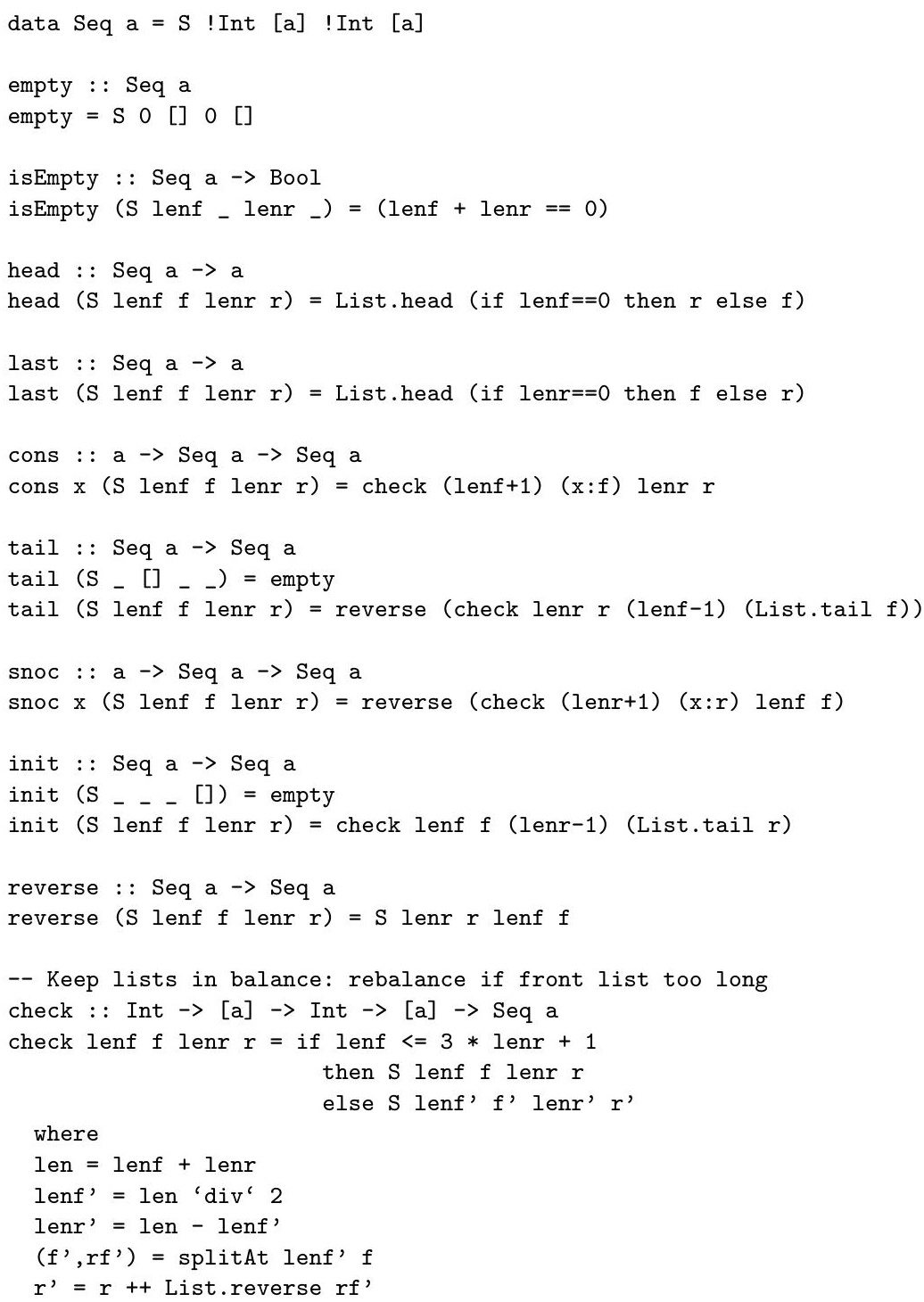

Fig. 4. The Banker's Implementation of Lazy Dequeues

regain for pretty a runtime that is only linear in the size of the input, we need dequeue functions that take only constant amortised time.

Figure 6 gives the banker's dequeue implementation of the sequence data type. A banker's dequeue is represented by two lists and their respective lengths. The first list holds the front elements and the other list holds the rear elements of the sequence in reverse order. An invariant requires that the lengths of the lists are not too far apart. A function check moves some elements from one list to the other, when addition or removal of an element threatens to invalidate the invariant. This rebalancing takes time linear in the length of the sequence, but rebalancing happens 
so seldomly that the amortised time of every function is constant. Okasaki gives more detailed explanations in his book [Okasaki 1998] ${ }^{6}$.

So we use the banker's dequeue for implementing the abstract sequence data type. However, we come across the same problem that we observed in the last section for the naive sequence implementation: the standard functions on banker's dequeues are too strict in the horizontal sequence hs. We cannot directly apply our previous solution: if the functions consTail, tailCons and initSnoc always copied the structure of the first to the second banker's dequeue, then we would not achieve our desired time complexity. However, the banker's dequeue implementation of tail demands only the first constructor of the first list representing the dequeue, the implementations of cons and snoc do not demand any part of the two lists, except when the two lists have to be rebalanced. Therefore the idea is that the linear time function copyListStructure is used only for rebalancing. Rebalancing takes linear time anyway.

The lazy Banker's dequeue implementation is given in Appendix C. This implementation cannot reuse the functions that operate on a single dequeue as the naive sequence implementation in Section 5 does: First, copyListStructure has to be used by the internal rebalancing function check. Second, the function check has to operate on both dequeues simultaneously. Whenever it rebalances the end position sequence, it also rebalances the horizontal sequence in exactly the opposite way using the structure of the end position sequence. Without this combined rebalancing corresponding dequeues es and hs would not have the same internal structure! There is more than one way to represent a sequence as a Banker's dequeue. ${ }^{7}$

The implementation of the function pretty of Figure 3 together with the sequence implementation of Appendix $\mathrm{C}$ give a bounded, linear time pretty printer.

\section{OVERFULL LINES}

We took Oppen's approach for formatting a group: a group is formatted horizontally if and only if it fits in the remaining space of the line. Unfortunately this approach may yield layouts with lines wider than the width limit, even when a fitting layout exists. A group that still fits on a line may be followed by further text without a separating line. Because there is no line, the text has to be added to the current line, even if it does not fit. Breaking the group might have avoided the problem.

The problem is solved by normalising the token list with respect to the following (confluent) rewriting rules before applying pretty:

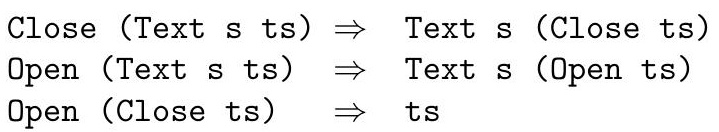

\footnotetext{
${ }^{6}$ The Haskell implementation in the book contains several mistakes. Also the implementation of Figure 6 uses reverse, taking advantage of symmetry, to simplify the implementation of check. ${ }^{7}$ For example, for most dequeues $d$ the dequeue tail (cons $42 d$ ) has a different representation from $d$. It is essential for the constant amortised runtime of all functions that there is more than one way to represent a sequence.
}

ACM Transactions on Programming Languages and Systems, Vol. TBD, No. TDB, Month Year. 
The normalised token list has the property that between a Close token and the next Text token there is always a Line token. ${ }^{8}$ In other words, the end of every group can be chosen to be the end of the line. Hence the aforementioned problem can no longer occur. With normalisation the pretty printer always produces a fitting layout if it exists, the pretty printer is optimal in Wadler's sense.

Rewriting only moves Text tokens in and out of groups. Therefore the set of lines "belonging" to each group, which are either all formatted as new lines or all as spaces, is unchanged. So normalisation leaves the set of strings denoted by a document unchanged. Only the representation of the document is changed, so that Oppen's formating criterium (possibly) selects a different set element as output.

In Appendix B normalisation is implemented by a linear traversal of the token list, which collects Open and Close tokens until the next Line token is reached.

\section{INDENTATION}

To complete the library we still have to implement the function nest. There are different interpretations of the expression nest $n$. In Wadler's library it increases the current left margin by $n$ columns whereas in Oppen's pretty printer (and other libraries) it sets the left margin to the current column position plus $n$. In Appendices $\mathrm{A}$ and $\mathrm{B}$ both variants are implemented. There are two new tokens:

data Tokens $=\ldots$

| OpenNest (Int $\rightarrow$ Int $\rightarrow$ Int) Tokens | CloseNest Tokens

The function of the first token takes the current margin and column to determine a new margin and the second token resets the margin to its previous value. An extended version of inter keeps a list of left margins and interprets the new tokens.

Alternatively, we could implement Wadler's variant as he does by a transformation which moves the indentation information into each Line token.

\section{COMPARISON WITH WADLER'S IMPLEMENTATION}

We followed Wadler in considering a document as equivalent to a set of strings. Like Hughes Wadler defines an ordering relation on lines: if two lines fit within the maximal width the longer one is better, otherwise the shorter one is better. The ordering on single lines is extended lexically to strings. Wadler's pretty printer outputs the string that is best with respect to the ordering.

Here we followed Oppen in formatting a group horizontal if and only if it fits on the current line. Because this specification refers directly to the construction of the document from combinators, it cannot be expressed in terms of the semantics of a document, the set of strings. However, we observed in Section 7 that token normalisation ensures that every group is followed by a potential end of line. Hence for a normalised token sequence Oppen's formatting criterium of filling a line with groups as far as possible without violating the width limit yields the output that is best with respect to Wadler's order. So the lazy dequeue pretty printer and Wadler's pretty printer yield the same output for any fully defined document. They

\footnotetext{
${ }^{8}$ Oppen [1980] states in his Section 2 that he assumes this property to hold for all input token sequences and hints at the end of Section 5 that other token sequences can be changed.
} 
are, however, not semantically equivalent. Although Wadler's implementation is bounded, it is still more strict, that is, it often requires more look-ahead. ${ }^{9}$

For example, with Wadler's library the Haskell interpreter Hugs computes

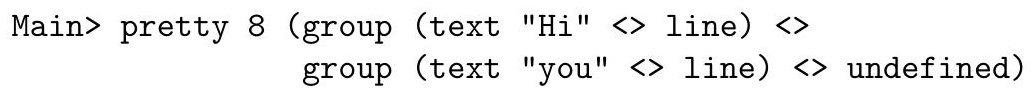

whereas with the lazy dequeue library it computes

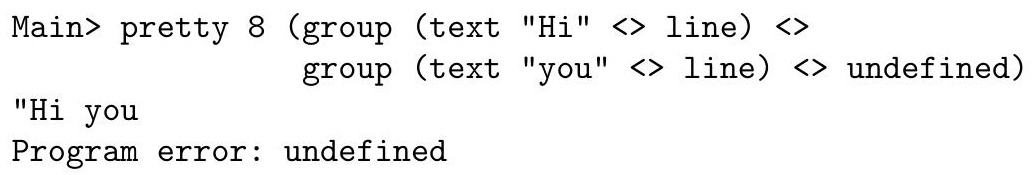

\section{IN PRACTICE}

Appendices $\mathrm{A}, \mathrm{B}$ and $\mathrm{C}$ give the full implementation of the lazy dequeue pretty printing library including token normalisation and two sorts of indentation.

Table I compares this library with the bounded but not linear implementation of Section 2.1, Wadler's library [Wadler 2003] and the one by Simon Peyton Jones based on John Hughes' [Peyton Jones 1997]. Note that the bounded implementation provides only basic pretty printing. In particular, it does not avoid overfull lines as discussed in Section 7 like all other libraries do. The table gives for different linewidths the time in seconds that is needed to format a sequence of 500 right-nested documents. The nested document (cf. Section 2.1) is generated by

doc $\mathrm{n}=$ if $\mathrm{n}==0$ then text " " else group (text "*" $<>$ line $<>\operatorname{doc}(\mathrm{n}-1)$ )

The test program for HPJ is

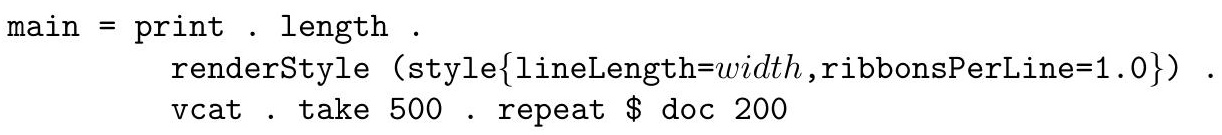

and for the other libraries it is the equivalent

main $=$ print . length . pretty width .

foldr1 ( $\backslash \mathrm{x}$ y $\rightarrow \mathrm{x}<>$ line $<>\mathrm{y}$ ). take 500 . repeat $\$$ doc 200

All programs were compiled with $\mathrm{GHC}^{10}$ version 6.2 with -02 and run on a $900 \mathrm{MHz}$ sparcv9 under Solaris.

The example clearly demonstrates that the runtimes of all implementations except our own depends on the line-width.

In practice the runtimes for line-widths of around 80 characters are of most interest. Because there is no "typical" document and the interface of HPJ is different, systematic comparisons are hard. In our experience the libraries provide similar

\footnotetext{
${ }^{9}$ Early on Wadler's implementation applies a flattening operation to the content of a group and the document following it. Thus information about the structure of the remaining document is lost. In contrast, the lazy dequeue implementation uses this information.

${ }^{10}$ The Glasgow Haskell compiler. http://www.haskell.org/ghc

ACM Transactions on Programming Languages and Systems, Vol. TBD, No. TDB, Month Year.
} 


\begin{tabular}{lrrrrrrr}
\hline \hline Line-width & 20 & 40 & 60 & 80 & 100 & 120 & 140 \\
\hline HPJ & 2.9 & 5.0 & 6.9 & 8.4 & 9.8 & 10.4 & 11.0 \\
Wadler & 1.1 & 1.9 & 2.5 & 3.2 & 3.8 & 4.1 & 4.4 \\
Bounded & 0.8 & 1.3 & 1.6 & 2.0 & 2.4 & 2.7 & 3.1 \\
Dequeue & 1.5 & 1.5 & 1.7 & 1.6 & 1.7 & 1.7 & 1.7 \\
\hline \hline
\end{tabular}

Table I. Runtimes in seconds for pretty printing a document with different line-width limits

runtimes, but for any two libraries there is a document where one library is up to 3-4 times faster than the other. Whereas the runtimes of HPJ vary most with changes of the document structure, those of Dequeue are the most stable.

\section{FINAL REMARKS}

We have developed a purely functional bounded pretty printer with the same time complexity as Oppen's imperative implementation. This proves that Oppen's algorithm can also be implemented purely functionally. We have seen that for a bounded pretty printer a dequeue is the natural choice for passing the maximal end position of a group forward along the token sequence to the point where it can be decided if the group is formatted horizontally or vertically. The key problem lies in passing the result of the decision backwards along the token sequence without jeopardising boundedness or the desired time complexity. For this purpose Oppen uses a mutable array; we use a second, synchronous, lazy dequeue.

Oppen's implementation consists of two parts which work together in a co-routine like fashion. So an explicitly concurrent version of the pretty printer seems natural. However, it would require additional explicit synchronisation to ensure that no process looked too far ahead, using unnecessary space for communication buffers. In the lazy dequeue implementation demand-driven evaluation ensures that the "process" computing the horizontal sequence never "goes" further ahead than the "output process" requires for formatting the next token.

The lazy dequeue implementation demonstrates two further points in algorithm design: First, defining a function recursively along the structure of the main data type (here a tree-structured document) may not lead to the best solution. We sometimes have to leave the limits of an implicit recursive control structure by making it explicit as data structure. A data structure can be replaced by a more flexible one (here a stack by a dequeue). ${ }^{11}$ Second, there are useful lazy variants of non-inductively defined abstract data structures such as dequeues.

A version of the pretty printing library with an extended interface is part of the distribution of the Haskell compiler nhc $98^{12}$ and has been used in various programs.

The lazy dequeue pretty printer is more complex than the non-linear implementation by Wadler and those given in Section 2. Numerous alternative implementations have been explored by me and other people. The failure to find a more simple linear and bounded implementation still does not settle the question whether such an implementation exists.

\footnotetext{
$\overline{{ }^{11} \mathrm{~A} \text { related }}$ well-known example is breadth-first traversal of a tree: Depth-first traversal can be implemented easily by direct recursion. However, instead of recursion we can also use a stack. Replacing the stack by a queue we obtain breadth-first traversal of a tree [Okasaki 2000].

${ }^{12}$ http://www.haskell.org/nhc98
} 
We developed the lazy dequeue pretty printer in a number of steps. Nonetheless we did not derive implementations from another by equational reasoning as Hughes and Wadler did. In fact, bounded and unbounded implementations have different strictness properties and hence are not semantically equivalent. We stepped several times from unbounded to bounded implementations and back. How can we prove the correctness of the development, so that it gives further insight into the problem and its solution?

\section{Acknowledgements}

I thank the referees and Colin Runciman for many suggestions for improving this paper. The work reported in this paper was partially supported by the Engineering and Physical Sciences Research Council of the United Kingdom under grant number GR/M81953.

\section{REFERENCES}

Azero, P. AND Swierstra, D. $1998 . \quad$ Optimal pretty-printing combinators. http://www.cs.uu.nl/groups/ST/Center/SoftwareDistributions.

BIRD, R. S. 1984. Using circular programs to eliminate multiple traversals of data. Acta Informatica 21, 239-250.

Hughes, J. 1986. A novel representation of lists and its application to the function "reverse". Information Processing Letters 22, 3, 141-144.

Hughes, J. 1995. The design of a pretty-printing library. In Advanced Functional Programming, J. Jeuring and E. Meijer, Eds. LNCS 925. Springer Verlag.

Okasaki, C. 1998. Purely Functional Data Structures. Cambridge University Press.

OKASAKI, C. 2000. Breadth-first numbering: lessons from a small exercise in algorithm design. In International Conference on Functional Programming. 131-136.

Oppen, D. C. 1980. Prettyprinting. ACM Transactions on Programming Languages and Systems 2, 4, 465-483.

Peyton Jones, S. L. 1997. A pretty printer library in Haskell. Part of the GHC distribution at http://www.haskell.org/ghc.

Peyton Jones, S. L., Ed. 2003. Haskell 98 Language and Libraries, The Revised Report. Cambridge University Press.

WAdLeR, P. 2003. A prettier printer. In The Fun of Programming. Palgrave Macmillan, Chapter $11,223-244$.

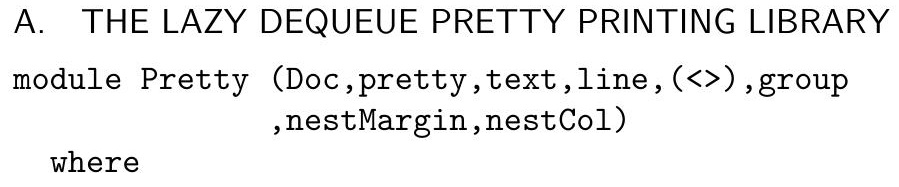




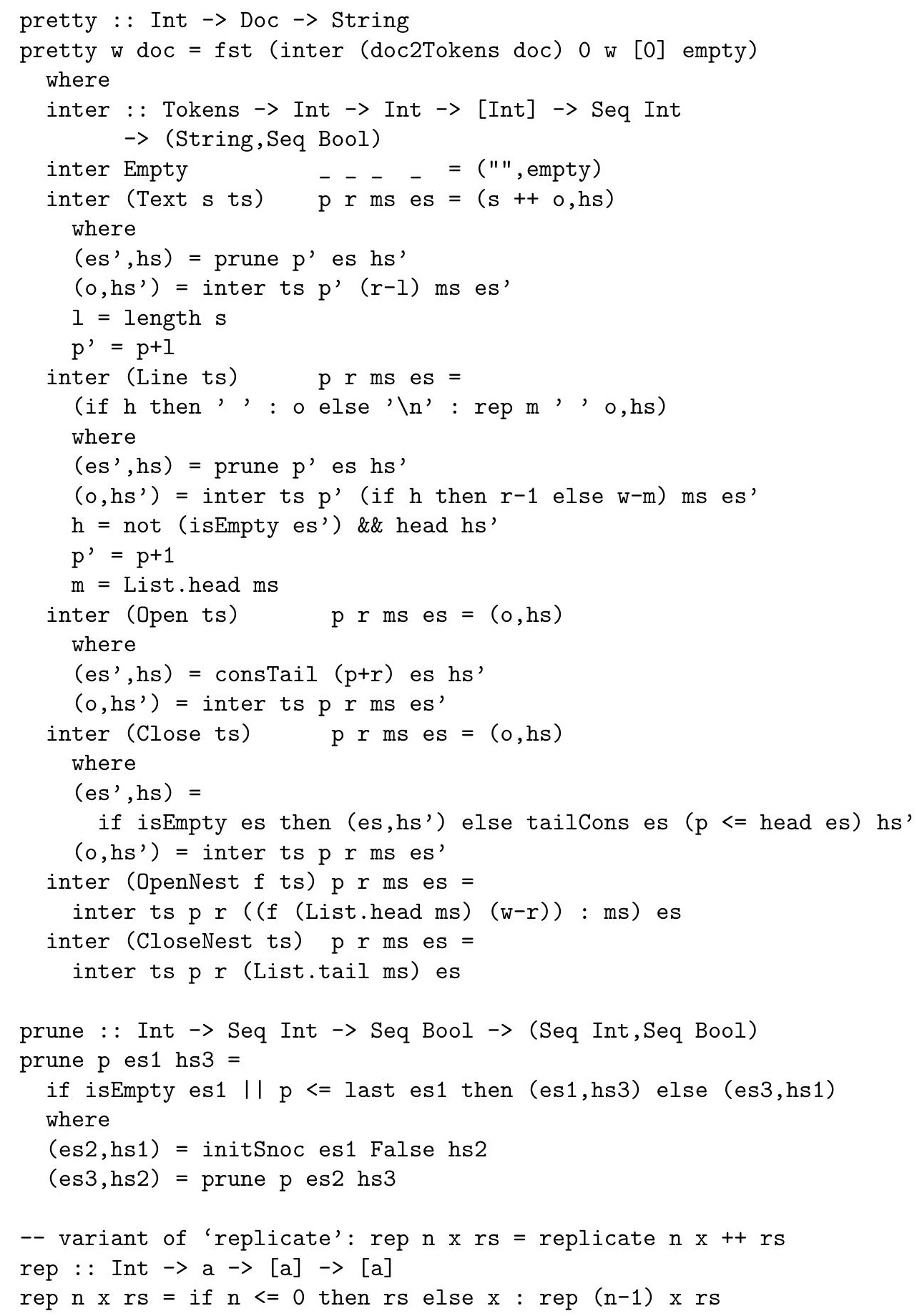




\section{B. IMPLEMENTATION OF THE DOCUMENT TYPE}

module Doc (text,line, (<>), group, nestMargin, nestCol, doc2Tokens, Doc , Tokens (Text, Line, Open, Close, OpenNest, CloseNest, Empty))

where

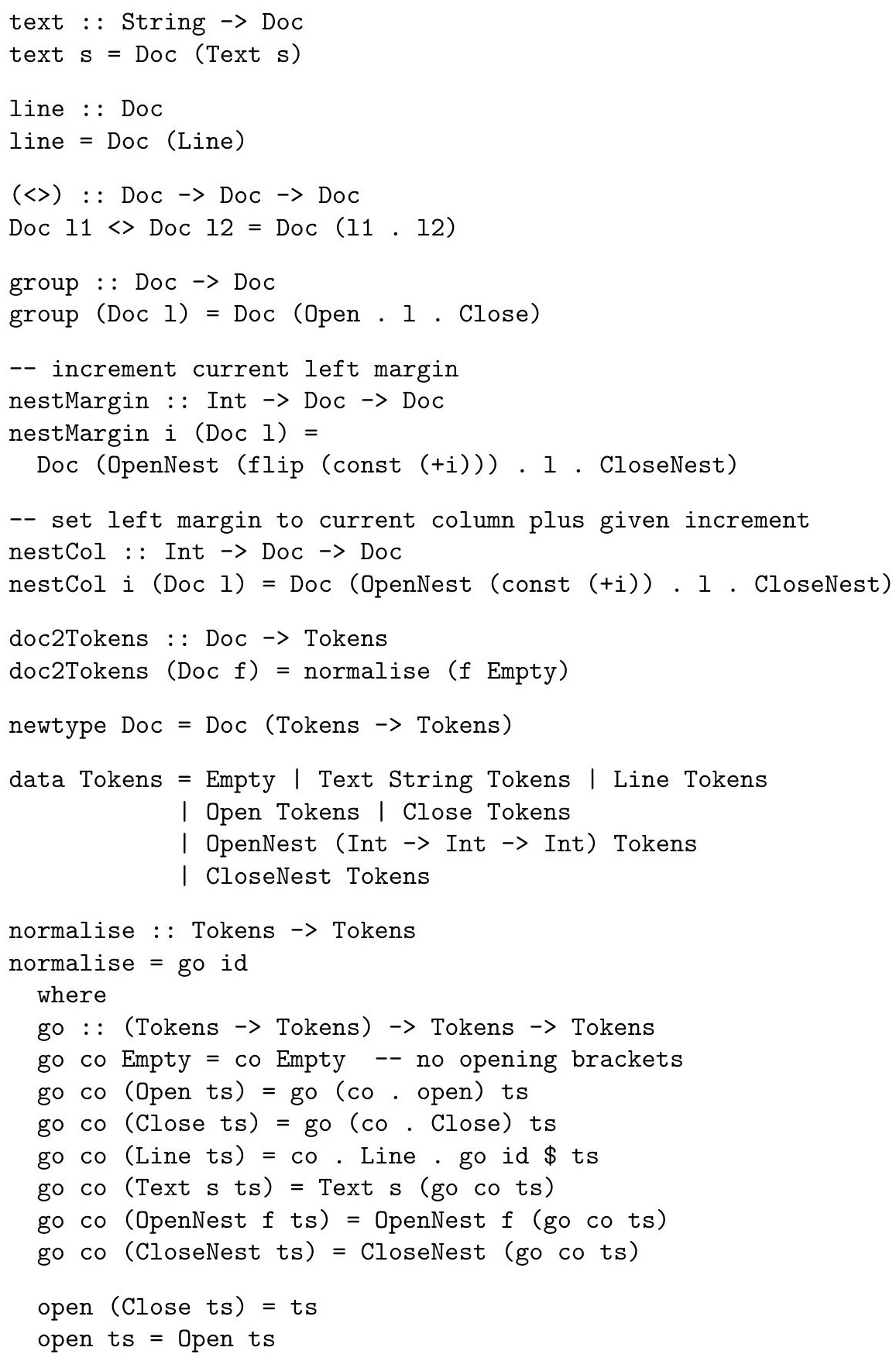

ACM Transactions on Programming Languages and Systems, Vol. TBD, No. TDB, Month Year. 


\section{IMPLEMENTATION OF EFFICIENT LAZY DEQUEUES}

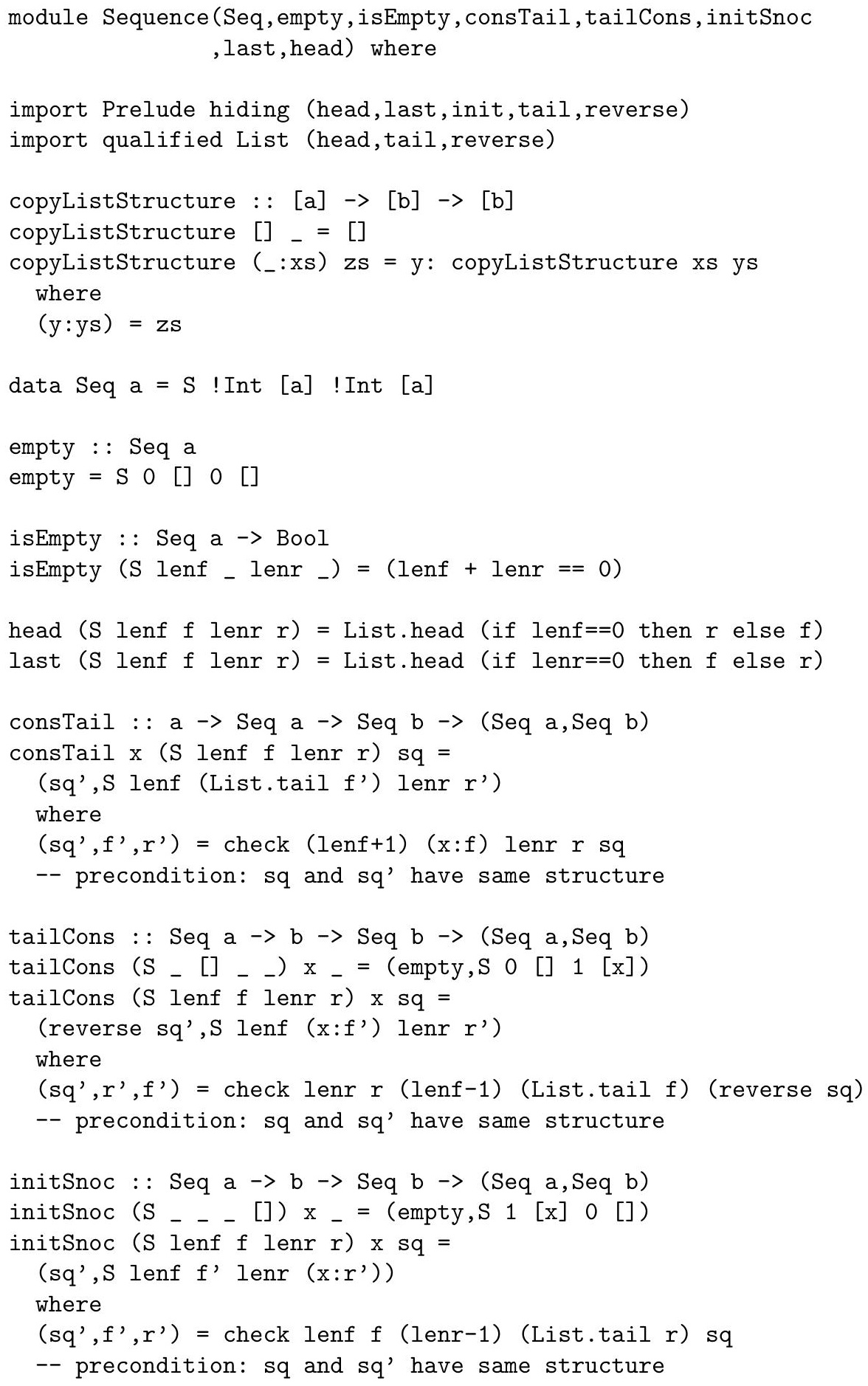


reverse : : Seq a $\rightarrow$ Seq a

reverse (S lenf $f$ lenr $r$ ) $=S$ lenr $r$ lenf $f$

-- Keep lists in balance: rebalance if front list too long check : : Int $\rightarrow[\mathrm{a}] \rightarrow$ Int $\rightarrow[\mathrm{a}] \rightarrow$ Seq $\mathrm{b} \rightarrow$ (Seq a, [b], [b])

check lenf $f$ lenr $\mathrm{r} s q=$ if lenf $<=3 *$ lenr +1

then (S lenf $f$ lenr $r, f 2, r 2$ )

else (S lenf' $f$ ' lenr' $r$ ',f2',r2')

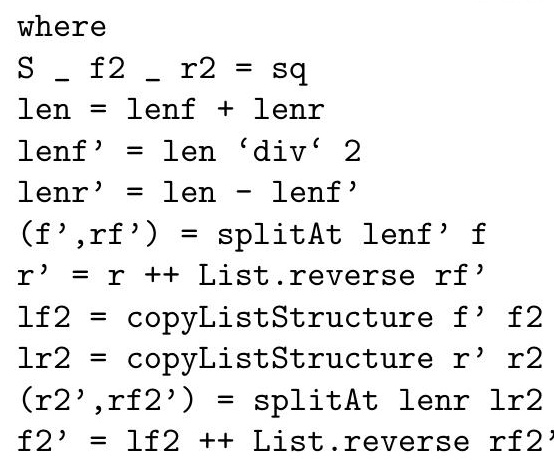


reverse : : Seq a $\rightarrow$ Seq a

reverse (S lenf $f$ lenr $r$ ) $=S$ lenr $r$ lenf $f$

-- Keep lists in balance: rebalance if front list too long check : : Int $\rightarrow[\mathrm{a}] \rightarrow$ Int $\rightarrow[\mathrm{a}] \rightarrow$ Seq $\mathrm{b} \rightarrow$ (Seq a, [b], [b])

check lenf $f$ lenr $\mathrm{r} s q=$ if lenf $<=3 *$ lenr +1

then (S lenf $f$ lenr $r, f 2, r 2$ )

else (S lenf' $f$ ' lenr' $r$ ',f2',r2')

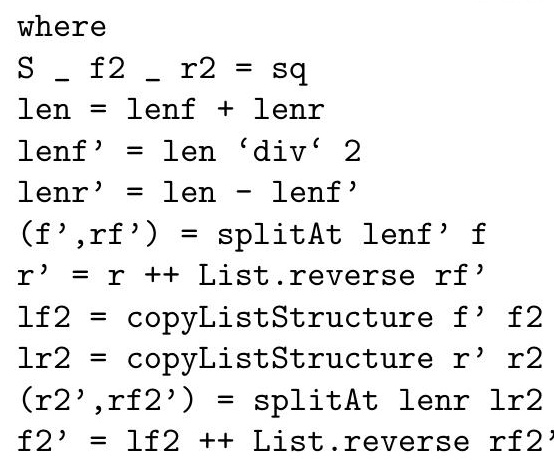

